\title{
Mosaic CREBBP mutation causes overlapping clinical features of Rubinstein-Taybi and Filippi syndromes
}

\author{
Tamar I de Vries ${ }^{1}$, Glen R Monroe ${ }^{1}$, Martine J van Belzen², Christian A van der Lans ${ }^{2}$, Sanne MC Savelberg ${ }^{1}$, \\ William G Newman ${ }^{3}$, Gijs van Haaften ${ }^{1}$, Rutger A Nievelstein ${ }^{4}$ and Mieke M van Haelst ${ }^{\star, 1}$
}

Rubinstein-Taybi syndrome (RTS, OMIM 180849) and Filippi syndrome (FLPIS, OMIM 272440) are both rare syndromes, with multiple congenital anomalies and intellectual deficit (MCA/ID). We present a patient with intellectual deficit, short stature, bilateral syndactyly of hands and feet, broad thumbs, ocular abnormalities, and dysmorphic facial features. These clinical features suggest both RTS and FLPIS. Initial DNA analysis of DNA isolated from blood did not identify variants to confirm either of these syndrome diagnoses. Whole-exome sequencing identified a homozygous variant in C9orf173, which was novel at the time of analysis. Further Sanger sequencing analysis of FLPIS cases tested negative for CKAP2L variants did not, however, reveal any further variants. Subsequent analysis using DNA isolated from buccal mucosa revealed a mosaic variant in $C R E B B P$. This report highlights the importance of excluding mosaic variants in patients with a strong but atypical clinical presentation of a MCA/ID syndrome if no disease-causing variants can be detected in DNA isolated from blood samples. As the striking syndactyly observed in the present case is typical for FLPIS, we suggest CREBBP analysis in saliva samples for FLPIS syndrome cases in which no causal CKAP2L variant is detected.

European Journal of Human Genetics (2016) 24, 1363-1366; doi:10.1038/ejhg.2016.14; published online 9 March 2016

\section{INTRODUCTION}

Rubinstein-Taybi syndrome (RTS, OMIM 180849) and Filippi syndrome (FLPIS, OMIM 272440) are both rare genetic disorder characterized by multiple congenital anomalies and intellectual deficit (MCA/ID). ${ }^{1,2}$

We present a patient with ID, dysmorphic features, syndactyly of hands and feet, and ocular features (microphthalmia and retinal coloboma). The differential diagnosis included RTS and FLPIS. Initial molecular diagnostic analysis, however, revealed no abnormalities in the RTS genes, CREBBP ${ }^{3}$ and EB300, ${ }^{4}$ nor in the FLPIS gene, CKAP2L. ${ }^{5}$ With whole-exome sequencing (WES) analysis, we identified a novel autosomal recessive deletion in C9orf173 that was novel at the time of analysis, causing a frameshift. However, no other FLPIS cases harbored variants in this gene. Subsequent analysis of DNA isolated from buccal mucosa showed a mosaic variant in CREBBP, confirming the atypical RTS diagnosis with clinical overlapping features of FLPIS.

\section{CLINICAL REPORT}

The proband is the third male child of non-consanguineous, healthy, Nepalese parents. He was born after a term pregnancy complicated by an episode of high maternal fever. The mother used non-specified medication during the pregnancy. Delivery was uncomplicated. After birth, a short stature was noticed. Unfortunately, anthropometric measurements are unavailable. Postnatally, he had feeding problems until 18 months of age. His developmental milestones were delayed. He did not walk independently until 4 years of age and has little to no speech development.
He was assessed by a clinical geneticist at the age of 11 years. At that time, he presented with severe ID, absent speech, combined conductive and sensorineural hearing loss, microphthalmia, retinal coloboma, hypotonia, hirsutism, and abnormal pigmentation of the skin. Height was $126.5 \mathrm{~cm}(-3.5 \mathrm{SD})$, weight $31.9 \mathrm{~kg}(-1.4 \mathrm{SD}$; weight to length, $+2 \mathrm{SD})$, and head circumference $51 \mathrm{~cm}(-1.7 \mathrm{SD})$. Dysmorphologic examination (Figure 1) showed ptosis, hypertelorism, upslanted short palpebral fissures, broad nose, thin lips, and teeth talon cusps. He had broad thumbs and halluces, and bilateral syndactyly of the second to fourth digits of the hands and feet. The syndactyly of the right hand has been surgically corrected.

At the age of 13 years and 10 months, height was $148.5 \mathrm{~cm}$ (less than $-2 \mathrm{SD}$ ), weight $53.2 \mathrm{~kg}(>2.5 \mathrm{SD})$, and the $\mathrm{OFC} 53 \mathrm{~cm}$ $(-1.5 \mathrm{SD})$. Radiologic examination at the age of 14 showed a relatively small neurocranium, enlarged sella turcica, luxation and deformation of the radial head, medial flattening of the distal radial and ulnar epiphysis, small and broad thumbs, brachydactyly of the distal phalanges, soft tissue syndactyly of the second to fourth finger (Figure 1c and f), and exostosis of the proximal fibula and tibia.

\section{LABORATORY INVESTIGATIONS AND RESULTS}

There were no indications for metabolic disorders in urine and blood samples. Karyotyping showed a normal male chromosome pattern $(46, \mathrm{XY})$ in blood and skin samples. Array CGH showed a paternal duplication of $11 \mathrm{q} 23.3$ and maternal deletion of 15q26.3. Sanger sequencing and MLPA analysis in blood samples revealed no likely disease-causing variants in the RTS genes, CREBBP and EP300.

\footnotetext{
${ }^{1}$ Department of Medical Genetics, University Medical Center Utrecht, Utrecht, The Netherlands; ${ }^{2}$ Department of Clinical Genetics, Leiden University Medical Center, Leiden, The Netherlands; ${ }^{3}$ Manchester Centre for Genomic Medicine, Institute of Human Development, University of Manchester, Manchester, UK; ${ }^{4}$ Department of Pediatric Radiology, University Medical Center Utrecht/Wilhelmina Children's Hospital, Utrecht, The Netherlands

*Correspondence: Dr MM van Haelst, Department of Medical Genetics, University Medical Center Utrecht, Lundlaan 6, Utrecht 3585EA, The Netherlands. Tel: +31 887553800; Fax: +31 887553801; E-mail: m.vanhaelst@umcutrecht.nl

Received 2 July 2015; revised 28 December 2015; accepted 3 January 2016; published online 9 March 2016
} 


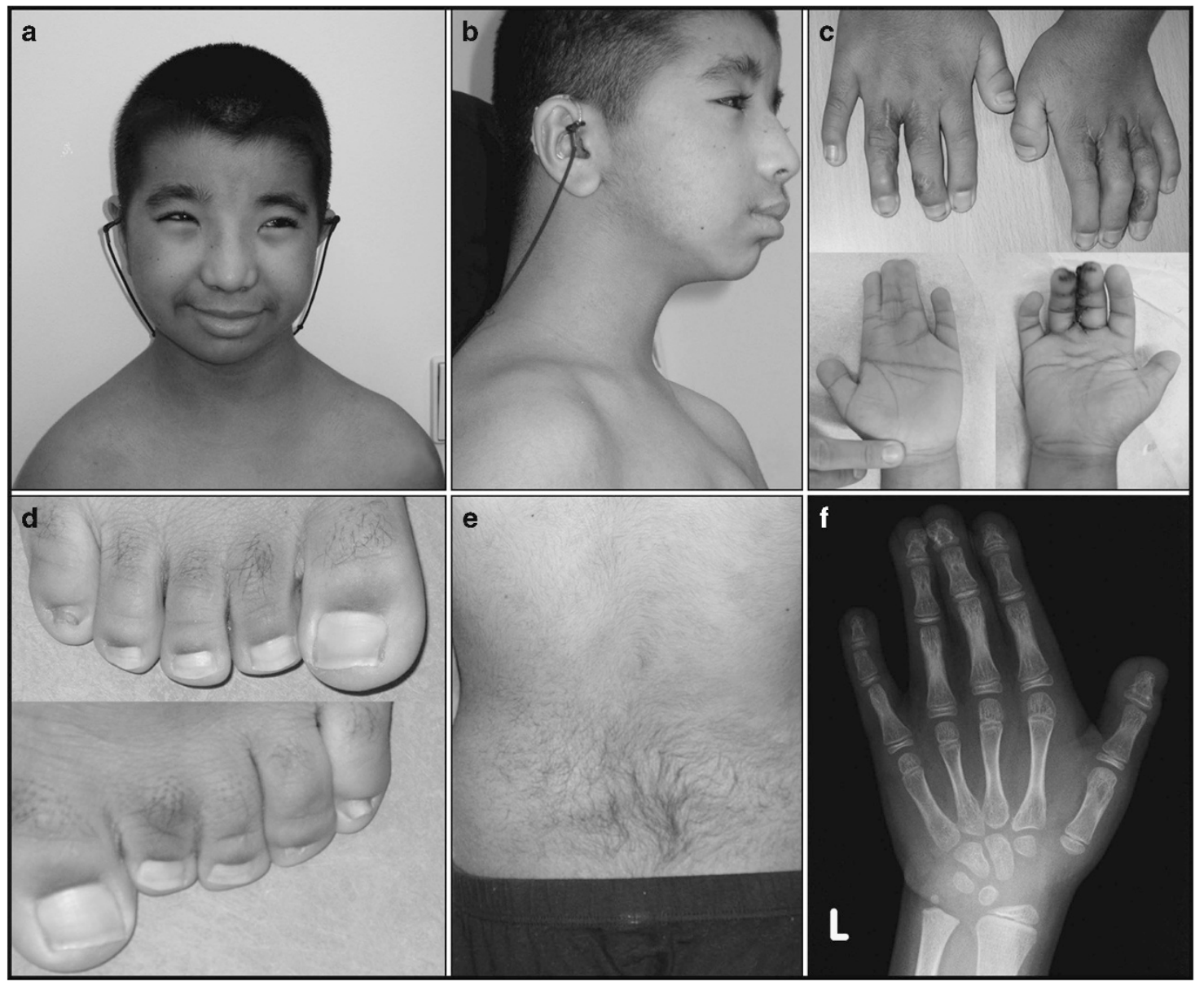

Figure 1 (a) Frontal photograph and (b) profile of the proband. Notice the hypertelorism and broad nose. (c) Hands: surgically corrected syndactyly of the third and fourth finger on the right hand, and syndactyly of the second, third, and fourth finger on the left. Note the broad thumbs. (d) Feet: syndactyly of the third and fourth toes. Note the broad first toes. (e) Back: note the hirsutism. (f) An X-ray of the left hand at the age of 10, showing brachydactyly of the distal phalanges and soft tissue syndactyly of the second to fourth finger. The full colour version of this figure is available at European Journal of Human Genetics online.

DNA of the patient and parents was isolated from blood, and fragment library preparation was performed according to an in-house protocol (Supplementary Text 1). WES of the patient and parents was done using an Illumina 2500 rapid run (Illumina, San Diego, CA, USA), and mean coverage was $105 \times$ with $93 \%$ covered $>20 \times$. After filtering on autosomal recessive, compound heterozygote and de novo inheritance models, the most likely candidate was a recessive $29 \mathrm{bp}$ deletion (NM_001256699.1:c.1072_1100del, p.(His358Glyfs*?)) in C9orf173 causing a frameshift. No functional studies for this gene have been reported in the literature. At the time of analysis, this variant was absent from multiple large variation databases, including the 1000 Genomes Project (http://www.1000genomes.org), NCBI dbSNP Build 137 for Human (http://www.ncbi.nlm.nih.gov/projects/ SNP), and the National Heart, Lung, and Blood Institute (NHLBI) GO Exome Sequencing Project Exome Variant Server (data release ESP6500; http://evs.gs.washington.edu/EVS). Unfortunately no Nepalese control samples were available. WES detected no candidate de novo variants.

In the meantime, a causal variant for FLPIS had been identified in CKAP2L. ${ }^{5}$ Variants in this gene were excluded in our patient. Sanger sequencing in three families with a clinical diagnosis of FLPIS that were tested negative for variants in CKAP2L yielded no variants in C9orf173. In the WES data of our patient, no variants were detected in the known causal genes for RTS, nor upon retrospective analysis in the causal gene CKAP2L for FLPIS. Meanwhile, we decided to exclude a mosaic form of RTS. Buccal mucosa was collected with the Oragene-DNA (OG-575; DNAGenotek, Ottawa, ON, Canada) kit for assisted collection. DNA isolation was performed according to manufacturer's instructions. Subsequently, Sanger sequencing of the complete coding regions of the RTS genes, CREBBP and EP300, was conducted in DNA isolated from buccal mucosa. A 3-bp deletion in CREBBP was found in mosaic form in exon 30: NM_004380.2:c.5039_5041delCCT, p. (Ser1680del), resulting in an in-frame deletion of a serine residue (Figure 2). This sequence variant has been submitted as CREBBP_00164 to the Leiden Open Variation Database (LOVD; http://chromium.lovd.nl/LOVD2/home.php?select_db $=$ CREBBP\&used_old_url) for patient \#0014986. This variant removes the recognition site for the restriction enzyme, BseRI, and restriction fragment length polymorphism analysis provided a secondary confirmation of the presence of the variant solely in buccal DNA. This particular variant lies within the functionally important histone acetyltransferase domain. This domain is highly conserved, and many different missense mutations in this domain have been detected in patients with RTS, ${ }^{6}$ in addition to two other in-frame amino acid deletions (LOVD). Protein modeling of other similar highly conserved amino acid variants within this region have been predicted to disrupt the domain structure and interfere with substrate interaction. ${ }^{7}$ As this variant lies in a highly conserved functional domain and is de novo, it is likely a 
Patient blood DNA Forward

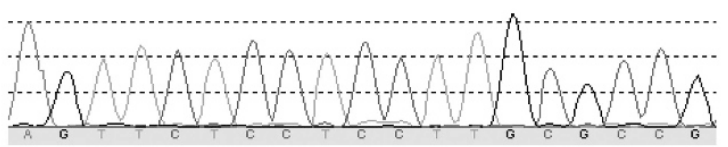

Reverse

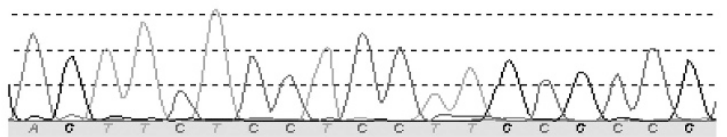

b Patient buccal mucosa DNA Forward

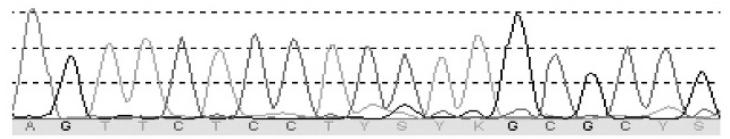

Reverse

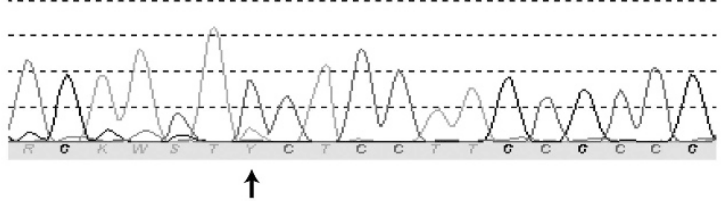

c

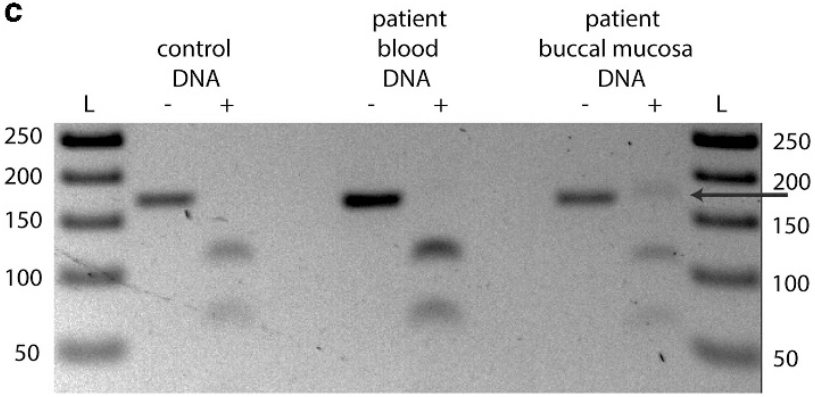

Figure 2 CREBBP mosaic variant in DNA isolated from buccal mucosa. The variant NM_004380.2:c.5039_5041delCCT, p.(Ser1680del) was not seen in DNA isolated from (a) blood, but was present in low-level mosaic form in DNA isolated from (b) buccal mucosa. Variant position is indicated with an arrow. (c) Following PCR with primers flanking the variant, restriction fragment length polymorphism analysis was used to confirm the mosaic deletion. Control DNA, DNA isolated from patient blood and buccal mucosa was PCR amplified, and digested with BseRI (+: BseRI treated). Ladder (L), undigested and digested control DNA (lanes 1/2), undigested and digested DNA isolated from patient blood (lanes 3/4), and undigested and digested DNA isolated from patient buccal mucosa (lanes 5/6). Only the DNA isolated from buccal mucosa after BseRI digestion has a PCR product that is undigested at $165 \mathrm{bp}$ (black arrow), due to the variant c.5039_5041delCCT removing the restriction enzyme recognition site. The full colour version of this figure is available at European Journal of Human Genetics online.

disease-causing variant. Upon reanalysis of the WES data, no reads for the alternate variant allele were present, even though the coverage was very high at $74 \times$. This mosaic variant was thus not detectable in DNA isolated from blood, but present in a low percentage in DNA isolated out from buccal mucosa.

\section{DISCUSSION}

The proband presented with ID, absence of speech, dysmorphic features, teeth talon cusps, coloboma, microphthalmia, bilateral
Table 1 Similarities and differences between RTS, ${ }^{1,8}$ FLPIS, $^{2,5}$ and our current case

\begin{tabular}{|c|c|c|c|}
\hline & RTS & FLPIS & Current case \\
\hline Intellectual deficit & + & + & + \\
\hline Speech impairment & - & + & + \\
\hline Microcephaly & + & + & - \\
\hline \multicolumn{4}{|l|}{ Short stature } \\
\hline Prenatal onset & - & + & + \\
\hline Postnatal growth retardation & + & + & + \\
\hline Syndactyly of hand and feet & + & + & + \\
\hline Cryptorchidism & + & + & - \\
\hline \multicolumn{4}{|l|}{ Dysmorphic features } \\
\hline Arched brows & + & - & - \\
\hline Downslanted palpebral fissures & + & - & - \\
\hline Upslanted palpebral fissures & - & - & + \\
\hline Hypertelorism & + & - & + \\
\hline Grimacing smile & + & - & - \\
\hline Beaked nose & + & - & - \\
\hline Prominent nasal bridge & + & + & + \\
\hline Wide, bulging forehead & - & + & - \\
\hline Talon cusps & + & - & + \\
\hline \multicolumn{4}{|l|}{ Ocular features } \\
\hline Coloboma & + & - & + \\
\hline Congenital cataract & + & - & - \\
\hline Lacrimal duct obstruction & + & - & - \\
\hline Congenital glaucoma & + & - & - \\
\hline Microphthalmia & + & - & + \\
\hline Hearing loss & + & - & + \\
\hline Seizures & - & + & - \\
\hline Hirsutism & + & + & + \\
\hline
\end{tabular}

Abbreviations: FLPIS, Filippi syndrome; RTS, Rubinstein-Taybi syndrome.

syndactyly of the second to fourth digits of hands and feet, and broad thumbs and halluces. The differential diagnosis included RTS and FLPIS. RTS is a MCA/ID syndrome, with a birth prevalence of 1 in 100 000-125 000, characterized by ID, microcephaly, postnatal growth deficiency, broad thumbs and halluces, and typical facial characteristics (Table 1). ${ }^{1,8}$ Other features include ocular abnormalities, including coloboma and microphthalmia, ${ }^{8}$ hearing loss, and congenital heart defects. Syndactyly of the hands is sometimes observed, usually between the third and fourth digits. ${ }^{9}$ Although it is not one of the diagnostic criteria, it appears that up to $20 \%$ of patients have a certain degree of syndactyly (Dr Rubinstein's database; Dr E Schorry, private communication). The presence of teeth talon cusps are specific for RTS and found in few other entities. ${ }^{1}$ RTS is caused by variants in CREBBP $(\sim 50-60 \% \text { of the cases) })^{3,10-12}$ and EP300 ( 3-8\%).,13 Although the features of the proband are consistent with RTS (Table 1), characteristic facial features, most notably the beaked nose and grimacing smile, are absent. Though the patient has a relatively small head size, there is no microcephaly. The upslanted short palpebral fissures were present in the father as well and are thus regarded as familial.

FLPIS is another MCA/ID syndrome, with currently 28 affected individuals from 20 families reported worldwide. ${ }^{2,5,14-16}$ The 
characteristic features include bilateral syndactyly of fingers and toes, microcephaly, ID with weak speech, short stature, bilateral cryptorchidism, and a characteristic face with prominent nasal bridge and bulging forehead. ${ }^{2,5}$ The syndrome has a variable phenotype, ${ }^{2}$ including seizures, visual disturbances, and ectodermal abnormalities (Table 1). ${ }^{2,5}$ Skeletal abnormalities have been reported on X-rays, including retarded bone age, brachymesophalangism (particularly of the fifth finger), hypoplasia of the radial heads with complete dislocation of the elbows, and bilateral synostosis of the carpal bones. Features in the present case consistent with FLPIS comprise ID, absent speech, short stature, bilateral syndactyly of hands and feet, and luxation and deformation of the radial head. Teeth talon cusps, retinal coloboma, and microphthalmia have not been previously described in FLPIS. Recently, CKAP2L was identified as the FLPIS gene. ${ }^{5}$

As no disease-causing variants in CREBBP, EP300, and CKAP2L were identified by Sanger sequencing on DNA isolated from blood, we performed a WES analysis. We identified an autosomal recessive deletion in C9orf173 that was absent from multiple large databases at the time of analysis. Unfortunately, no link could be made to gene function and other FLPIS cases negatively tested for CKAP2L variants showed no variants in this gene. The variant was later identified in The 1000 Genomes Project as rs544669429 and present at a minor allele frequency of $0.0044(0.44 \%)$ and most likely a neutral variant.

We subsequently identified a likely disease-causing variant in CREBBP by Sanger sequencing in DNA isolated from buccal mucosa. The particular 3-bp variant found in our patient has been described once before in a RTS patient, albeit not in mosaic form. ${ }^{12}$ This patient's phenotype is similar to ours, with delayed walking, broad thumbs, syndactyly of hands and feet, feeding problems, and retinal coloboma. Unlike the present case, this patient also has a bicuspid aorta valve and typical RTS facial features with a beaked nose and downward slanting palpebral fissures (Dr E Schorry, private communication).

Mosaic variants in CREBBP have been described previously in RTS. ${ }^{17-19}$ However, previous mosaic variants involving CREBBP have been identified in peripheral blood samples. ${ }^{17-19}$ The current case is the first case in which the variant was detected solely in buccal mucosa and not in blood samples. Mosaic de novo variants absent from blood samples have also been described in other conditions. Recently, it was reported that mosaic variants were identified in saliva-derived DNA in patients with a clinical diagnosis of Cornelia de Lange syndrome that were tested negative for variants in peripheral blood. ${ }^{20}$ With the present report, we further support the suggestion that patients presenting with a de novo MCA/ID syndrome tested negative for suggested gene variants in peripheral blood should also have this specific molecular analysis in DNA isolated from other tissues. This might prevent unnecessary extensive diagnostic analysis for other MCA/ID syndromes. It is possible that there is underdiagnosis of RTS in mild/atypical cases due to mosaicism.

As far as we are aware, a clinical overlap between RTS and FLPIS has not been previously described. In one reported case, FLPIS and RTS were considered in the differential diagnosis, but FLPIS was quickly dismissed. ${ }^{21}$ Our patient, however, shows overlapping features of both syndromes (Table 1). We propose that RTS and FLPIS should be considered in their respective differential diagnoses.
Although the underlying gene defect has recently been identified in FLPIS, there are still FLPIS cases that cannot be explained by CKAP2L variants. Sequence analysis of CREBBP in DNA isolated from buccal mucosa could be indicated in those cases.

\section{CONFLICT OF INTEREST}

The authors declare no conflict of interest.

\section{ACKNOWLEDGEMENTS}

We would like to thank Dr Elizabeth Schorry for the correspondence. Tamar de Vries is a medical student participating in the Honour's program of the Faculty of Medicine, UMC Utrecht.

1 Hennekam RC: Rubinstein-Taybi syndrome. Eur J Hum Genet 2006; 14: 981-985.

2 Battaglia A, Filippi T, Pusceddu S, Williams CA: Filippi syndrome: further clinical characterization. Am J Med Genet A 2008; 146A: 1848-1852.

3 Petrij F, Giles RH, Dauwerse HG et al: Rubinstein-Taybi syndrome caused by mutations in the transcriptional co-activator CBP. Nature 1995; 376: 348-351.

4 Roelfsema JH, White SJ, Ariyürek $Y$ et al: Genetic heterogeneity in Rubinstein-Taybi syndrome: mutations in both the CBP and EP300 genes cause disease. Am J Hum Genet 2005; 76: 572-580.

5 Hussain MS, Battaglia A, Szczepanski S et al: Mutations in CKAP2L, the human homolog of the mouse Radmis gene, cause Filippi Syndrome. Am J Hum Genet. 2014; 95: 622-632.

6 Roelfsema JH, Peters DJ: Rubinstein-Taybi syndrome: clinical and molecular overview. Expert Rev Mol Med 2007; 9: 1-16.

7 Mullighan CG, Zhang J, Kasper LH et al: CREBBP mutations in relapsed acute lymphoblastic leukaemia. Nature 2011; 471: 235-239.

8 Van Genderen MM, Kinds GF, Riemslag FC, Hennekam RC: Ocular features in Rubinstein-Taybi syndrome: investigation of 24 patients and review of the literature. Br J Ophthalmol 2000; 84: 1177-1184.

9 Hutchinson DT, Sullivan R: Rubinstein-Taybi Syndrome. J Hand Surg Am 2015; 40: 1711-1712.

10 Coupry I, Roudaut C, Stef M et al: Molecular analysis of the CBP gene in 60 patients with Rubinstein-Taybi syndrome. J Med Genet 2002; 39: 415-421.

11 Bartsch 0, Schmidt S, Richter M et al: DNA sequencing of CREBBP demonstrates mutations in $56 \%$ of patients with Rubinstein-Taybi syndrome (RSTS) and in another patient with incomplete RSTS. Hum Genet 2005; 117: 485-493.

12 Schorry EK, Keddache M, Lanphear N et al: Genotype-phenotype correlations in Rubinstein-Taybi syndrome. Am J Med Genet A 2008; 146A: 2512-5219.

13 Negri G, Milani D, Colapietro P et al: Clinical and molecular characterization of Rubinstein-Taybi syndrome patients carrying distinct novel mutations of the EP300 gene. Clin Genet 2015; 87: 148-154.

14 Sharif S, Donnai D: Filippi syndrome: two cases with ectodermal features, expanding the phenotype. Clin Dysmorphol 2004; 13: 221-226.

15 Cabala M, Stevens SJ, Smigiel R: A case of Filippi syndrome with atypical limb defects in a 3-year-old boy and a review of the literature. Clin Dysmorphol 2013; 22: 146-148.

16 Sandhu M, Malik P, Saha R: Multiple dental and skeletal abnormalities in an individual with filippi syndrome. Case Rep Dent 2013; 2013: 845405.

17 Gervasini C, Castronovo P, Bentivegna A et al: High frequency of mosaic CREBBP deletions in Rubinstein-Taybi syndrome patients and mapping of somatic and germ-line breakpoints. Genomics 2007; 90: 567-573.

18 Bartsch O, Kress W, Kempf O, Lechno S, Haaf T, Zechner U: Inheritance and variable expression in Rubinstein-Taybi syndrome. Am J Med Genet A 2010; 152A: 2254-2261.

19 Chiang PW, Lee NC, Chien N, Hwu WL, Spector E, Tsai AC: Somatic and germ-line mosaicism in Rubinstein-Taybi syndrome. Am J Med Genet A 2009; 149A: 1463-1467.

20 Ansari M, Poke G, Ferry Q et al: Genetic heterogeneity in Cornelia de Lange syndrome (CdLS) and CdLS-like phenotypes with observed and predicted levels of mosaicism. J Med Genet 2014; 51: 659-668.

21 Ravel A, Chouery E, Stora S et al: How many entities exist for the spectrum of disorders associated with brachydactyly, syndactyly, short stature, microcephaly, and intellectual disability? Am J Med Genet A 2011; 155A: 880-884. 